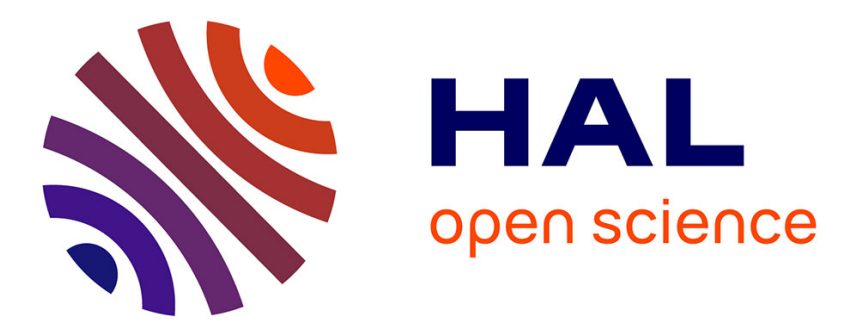

\title{
Asymmetrical stripline based method for retrieving the electromagnetic properties of metamaterials
}

Sandra Gomez, Patrick Queffelec, Alexis Chevalier, Anne-Claude Tarot, Ala Sharaiha

\section{- To cite this version:}

Sandra Gomez, Patrick Queffelec, Alexis Chevalier, Anne-Claude Tarot, Ala Sharaiha. Asymmetrical stripline based method for retrieving the electromagnetic properties of metamaterials. Journal of Applied Physics, 2013, Vol. 113 (024912), pp.024912-1-6. hal-00801289

HAL Id: hal-00801289

https://hal.univ-brest.fr/hal-00801289

Submitted on 15 Mar 2013

HAL is a multi-disciplinary open access archive for the deposit and dissemination of scientific research documents, whether they are published or not. The documents may come from teaching and research institutions in France or abroad, or from public or private research centers.
L'archive ouverte pluridisciplinaire HAL, est destinée au dépôt et à la diffusion de documents scientifiques de niveau recherche, publiés ou non, émanant des établissements d'enseignement et de recherche français ou étrangers, des laboratoires publics ou privés. 


\section{AIP Appilied Physics}

\section{Asymmetrical stripline based method for retrieving the electromagnetic properties of metamaterials}

S. Gómez, P. Quéffélec, A. Chevalier, A. C. Tarot, and A. Sharaiha

Citation: J. Appl. Phys. 113, 024912 (2013); doi: 10.1063/1.4775721

View online: http://dx.doi.org/10.1063/1.4775721

View Table of Contents: http://jap.aip.org/resource/1/JAPIAU/v113/i2

Published by the American Institute of Physics.

\section{Related Articles}

Wood anomaly transmission enhancement in fishnet-based metamaterials at terahertz frequencies J. Appl. Phys. 112, 124509 (2012)

Micromachined switchable metamaterial with dual resonance Appl. Phys. Lett. 101, 151902 (2012)

Research on pass band with negative phase velocity in tubular acoustic metamaterial J. Appl. Phys. 112, 053523 (2012)

Control of reflectance and transmittance in scattering and curvilinear hyperbolic metamaterials Appl. Phys. Lett. 101, 091105 (2012)

A fast Fourier transform implementation of the Kramers-Kronig relations: Application to anomalous and left handed propagation

AlP Advances 2, 032144 (2012)

\section{Additional information on J. Appl. Phys.}

Journal Homepage: http://jap.aip.org/

Journal Information: http://jap.aip.org/about/about_the_journal

Top downloads: http://jap.aip.org/features/most_downloaded

Information for Authors: http://jap.aip.org/authors

\section{ADVERTISEMENT}

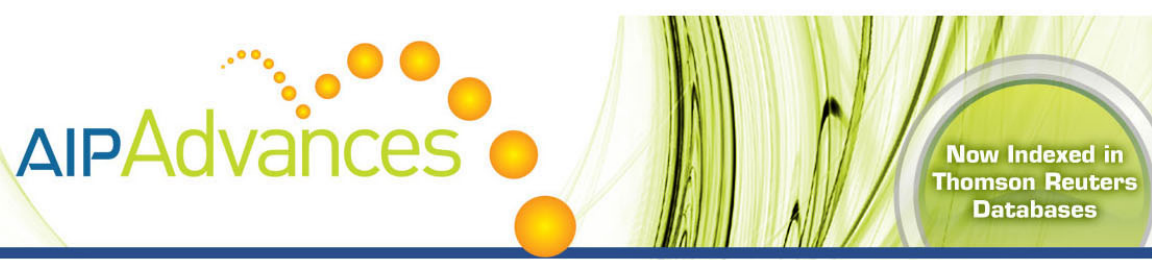

\section{Explore AIP's open access journal: Rapid publication \\ - Article-level metrics \\ Post-publication rating and commenting}




\title{
Asymmetrical stripline based method for retrieving the electromagnetic properties of metamaterials
}

\author{
S. Gómez, ${ }^{1, a)}$ P. Quéffélec, ${ }^{1}$ A. Chevalier, ${ }^{1}$ A. C. Tarot ${ }^{2}$ and A. Sharaiha ${ }^{2}$ \\ ${ }^{1}$ Laboratoire des Sciences et Techniques de l'information, de la Communication et de la Connaissance \\ (Lab-STICC) UMR CNRS 6285, European University of Brittany-UBO, CS 93837, 29238 Brest, France \\ ${ }^{2}$ Institute of Electronics and Telecommunications of Rennes (IETR) UMR CNRS 6164, University of Rennes 1, \\ 35042 Rennes Cedex, France
}

(Received 6 September 2012; accepted 20 December 2012; published online 14 January 2013)

\begin{abstract}
An experimental characterization technique for determining the electromagnetic properties of metamaterials in the microwave frequency band is presented. The method consists in measuring the S-parameters of an asymmetrical stripline partially filled with the sample to be characterized. For retrieving the characteristic parameters, two different approaches based on quasi-static approximations are compared. This measurement cell allows an "in situ" characterization because it reproduces the electromagnetic environment frequently found in planar technologies for microwave applications of metamaterials. The whole sample is uniformly excited in magnitude and direction by the interacting electromagnetic field which guarantees representativeness of extracted permittivity and permeability and avoids possible inconsistencies due to anisotropy and heterogeneity of metamaterials. (C) 2013 American Institute of Physics. [http://dx.doi.org/10.1063/1.4775721]
\end{abstract}

\section{INTRODUCTION}

Specific constitution of metamaterials imposes considering certain conditions in order to characterize their electromagnetic behavior. Particular attention must be given to some properties like shape anisotropy, heterogeneity and dispersion, which are related to their composite character (typically arrays of metallic inclusions inside a dielectric substrate). In the first place, anisotropy entails that the response of the material changes depending on the direction of the incident field which means that permittivity and permeability should be represented by tensor quantities. In order to determine without ambiguity one component of the permittivity and permeability tensors, all the unit cells constituting the sample must be excited by the same polarization and magnitude of the incident electromagnetic field. In the second place, heterogeneity in metamaterials implies that the method used for extracting characteristic parameters must consider a minimum number of inclusions in order to get the asymptotic behavior of the metamaterial close to the ideal case with an infinite number of inclusions.

Existing experimental techniques for extracting electromagnetic properties of metamaterials are based on the measurement of scattering parameters (S-parameters) of a particular guided or unguided structure containing a sample of material. Then, permittivity and permeability are retrieved by an electromagnetic analysis of the structure which generally includes an inversion procedure. Among guided methods, the most used are resonators, ${ }^{1}$ waveguides ${ }^{2,3}$ and planar transmission lines. ${ }^{4}$ Even though having good sensitivity, resonating structures are not well suited for characterizing materials exhibiting dispersive properties. In waveguide based techniques, the particular electromagnetic field pattern of propagation modes implies that all the inclusions are not

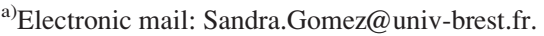

homogeneously excited. In the general case of stripline techniques, the sample fills the entire cross section of the line which is illuminated by a TEM mode. This configuration entails that inclusions which are far from the strip conductor will be excited by different polarization of the electromagnetic field than inclusions under the strip. Depending on the location inside the line, additional correction due to anisotropy should be applied. Finally, free space methods ${ }^{5,6}$ guarantee the correct excitation by the incident field (planar wave), but at relative low frequencies (from $10 \mathrm{MHz}$ up to $6 \mathrm{GHz}$ ) the considerable size of the samples and required set up makes their implementation very difficult and expensive.

This work describes an experimental method for extracting electromagnetic parameters of metamaterials between $10 \mathrm{MHz}$ and $6 \mathrm{GHz}$ considering the two characteristics mentioned before: heterogeneity and anisotropy. The measurement cell consists in an asymmetrical stripline originally developed for characterizing magnetic materials. ${ }^{7,8}$ The main advantage of this method is that orientation and distribution of the electromagnetic field seen by all the inclusions is homogeneous. This particular setup also reproduces an environment very close to that met in practice for typical planar applications (antennas for example). The article will be organized as follows: after presenting the method and their advantages for characterizing metamaterials, two retrieval procedures will be exposed and experimental results using a metasolenoid type metamaterial will be compared with simulated data.

\section{STRIPLINE METHOD}

The asymmetrical stripline used in this characterization technique is made up of a central conductor enclosed by two ground planes. The conductor is closer to the inferior plane in order to concentrate the most part of the energy in the region where the sample is set as shown in Fig. 1. The 


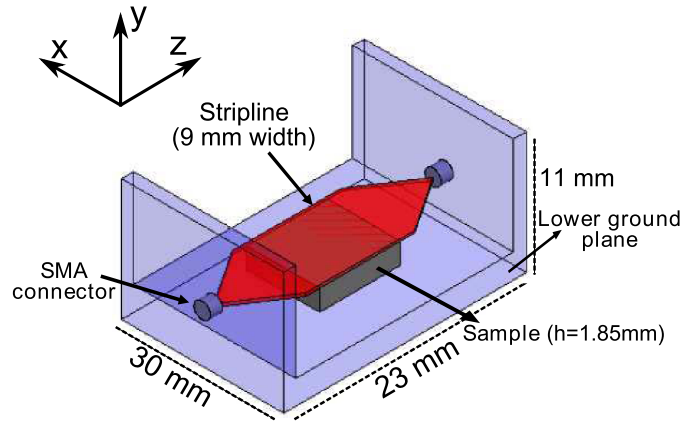

FIG. 1. Drawing of the stripline measurement cell (superior ground plane is removed).

dimensions of the microwave structure are designed to have $50 \Omega$ characteristic impedance. Conductors's width is $9 \mathrm{~mm}$, height between lower ground plane and central strip is $1.85 \mathrm{~mm}$, and height from strip to superior ground plane is $9 \mathrm{~mm}$. The total length of the cell is $23 \mathrm{~mm}$ and its width is $30 \mathrm{~mm}$.

A TEM mode is propagated through the empty cell but in the region loaded by the material the propagated mode is of quasi-TEM type due to the inhomogeneous character of the cross-section. Just below the conductor the configuration of the electromagnetic field becomes homogeneous in magnitude and direction, being the electric field parallel to the inclusions and the magnetic field perpendicular to the rings of the metamaterial (Fig. 2). The principle of the method consists in measuring the scattering parameters (S-parameters) of the stripline loaded by the sample under test using a Vectorial Network Analyzer. Then, characteristic parameters (complex permittivity $\epsilon$ and permeability $\mu$ ) are retrieved using analytical procedures. Data processing software is divided in two sections: an electromagnetic analysis of the cell (direct problem) and an inverse process to extract the electromagnetic parameters of the sample.

Asymmetrical character of the line, which concentrates almost all the energy in the lowest region of the cell, allows us to approach its overall behavior as a microstrip line. Nevertheless, when measuring metamaterials, sample's width must be shorter than the conductor's one to insure the same field distribution for all inclusions. In these experimental conditions, the microstrip assumption is no longer valid and the propagation structure becomes more complex.

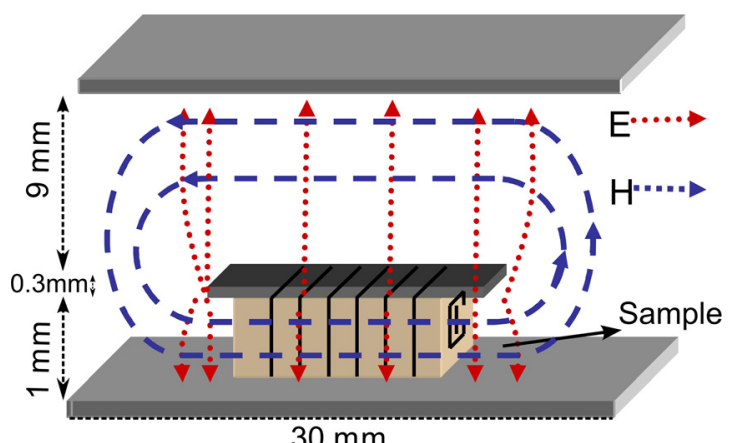

FIG. 2. Electromagnetic field pattern in the region of the line loaded by the sample to be characterized.
Two kind of electromagnetic analysis had been tested for extracting the constitutive parameters of a metamaterial from the measured S-parameters of the cell. They are both based on the quasi-static approximation and their main difference is the way they consider the heterogeneous nature of the transversal section of the loaded stripline, as it will be discussed in Sec. III.

\section{DATA PROCESSING PROCEDURE}

\section{A. Variational approach}

The first type of analysis that will be presented is based on variational theory. ${ }^{9}$ In this case, the transversal section of the cell is divided in horizontal layers of air and material distributed as shown in Fig. 3(a) (note that for implementing this approach it is necessary to assume that the sample fills all the width of the stripline). Using quasi-static hypothesis, we can find relationships between effective parameters of the structure $\epsilon_{\text {eff }}^{\text {th }}, \mu_{\text {eff }}^{\text {th }}$ and its equivalent inductances and capacitances,

$$
\mu_{e f f}^{t h}=\frac{L_{\left(\mu_{\text {meta }}\right)}}{L_{0}} \quad \text { and } \quad \epsilon_{e f f}^{t h}=\frac{C_{\left(\epsilon_{\text {meta }}\right)}}{C_{0}}
$$

where $C_{\left(\epsilon_{\text {meta }}\right)}$ is the linear capacitance, $L_{\left(\mu_{\text {meta }}\right)}$ is the linear inductance of the loaded structure, and $C_{0}, L_{0}$ correspond to the same parameters but for the structure without the sample to be characterized (empty cell).

The Green's functions are used for solving the characteristic equations of a transmission line composed of different layers and determining the values of $L_{\left(\mu_{\text {meta }}\right)}$ and $C_{\left(\epsilon_{\text {meta }}\right)}$. This procedure (named-“direct problem") allows us to calculate the theoretical effective permittivity and permeability of an equivalent material filling the cross section of the cell $\left(\epsilon_{e f f}^{\text {th }}, \mu_{e f f}^{\text {th }}\right)$, which are functions of intrinsic permittivity $\epsilon_{\text {meta }}$ and permeability $\mu_{\text {meta }}$ of the metamaterial.

The second stage of the procedure (inverse problem) consists in retrieving the values of $\epsilon_{\text {eff }}^{\text {meas }}$ and $\mu_{\text {eff }}^{\text {meas }}$ from the measured $S$ parameters. In order to do that, the composite structure should be homogenized to be treated as a conventional homogeneous transmission line filled by an unique material with effective permittivity and permeability (Fig. 3(b)). Then, conventional retrieval procedure NicolsonRoss-Weir (NRW) ${ }^{10}$ can be used. Once we obtain the values of $\epsilon_{\text {eff }}^{\text {meas }}$ and $\mu_{\text {eff }}^{\text {meas }}$ from measurement, an optimization procedure is implemented for matching theoretical and measured effective permittivity and permeability. Intrinsic values

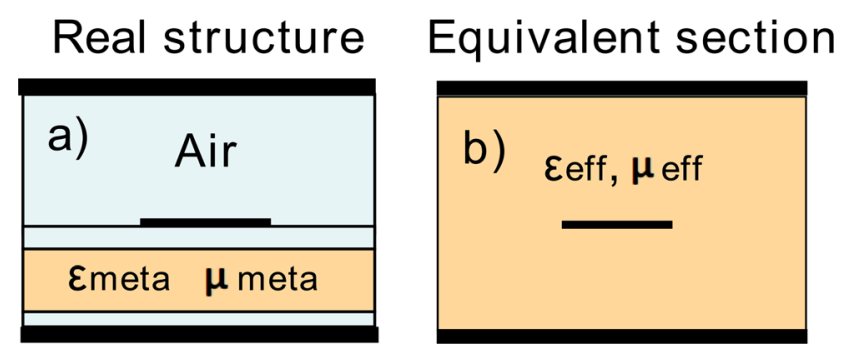

FIG. 3. Homogenization of the cross section of the cell-variational approach. 
$\left(\epsilon_{\text {meta }}\right.$ and $\left.\mu_{\text {meta }}\right)$ are taken as optimization variables. We developed a home code using the software MathWorks ${ }^{\circledR}$ MATLAB and a routine for solving non-linear equations based on Gauss-Newton method. ${ }^{11}$

\section{B. Transmission line theory based approach}

The second approach for retrieving the complex parameters of a material set inside the measurement cell is based on the transmission line theory. Assuming that the most part of the electromagnetic energy is concentrated in the region below the central strip, we consider only the region located between the strip and the lowest ground plane (Fig. 4(a)). In terms of electromagnetic analysis, resultant section is an equivalent two conductors transmission line shown in Fig. 4(b). Perfect electric conductor (PEC) walls represent central strip and inferior ground plane, while perfect magnetic conductor (PMC) walls are used as boundary conditions at the edge of the strip considering the symmetry of the field pattern related to the quasi-TEM propagated mode.

As for the variational approach, transversal section is composed of different layers (air and metamaterial) but, in this case, the width of the metamaterial sample do not exceeds the width of the central strip and we can even consider air at each side of the sample. Direct problem consists in solving the Telegrapher equations of the transmission line, giving expressions of $\mathrm{L}$ and $\mathrm{C}$ in terms of the dimensions, permittivity, and permeability of the different regions. Then, S-parameters of the structure are expressed in terms of transmission and reflection coefficients, which depend on the impedance $Z$ and the propagation constant $\gamma$ of the line. Finally, direct problem gave us the S-parameters of the cell as functions of permittivity and permeability of the different materials.

In order to inverse the electromagnetic analysis (inverse problem), expressions of the permittivity and the permeability of the metamaterial are given in function of the measured $\mathrm{S}$-parameters using a method comparable to the NRW procedure. ${ }^{10}$ In comparison with the previous electromagnetic analysis (variational procedure), the main advantage of the theoretical approach based on the transmission line theory lies in the fact that analytical relations can be established to express the permittivity and the permeability of the metamaterial to be characterized as functions of the measured S-parameters of the test device. ${ }^{8}$ The analytical expressions (2), (3) that directly relate S-parameters and constitutive parameters of metamaterials are the following:

\section{a) Real structure}

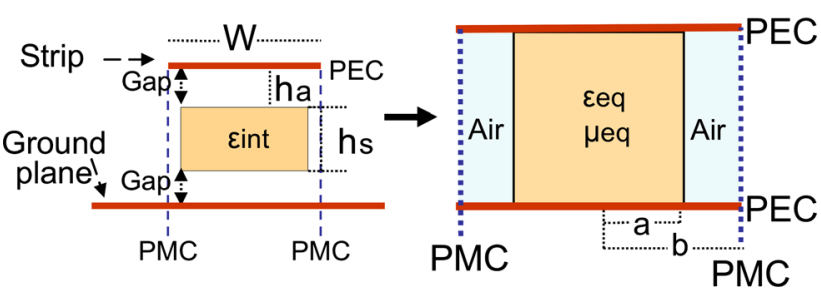

FIG. 4. Modelisation of the inferior loaded cross section of the stripline with equivalent two conductors line.

$$
\begin{gathered}
\mu=\frac{a Z_{0} 2 \gamma(1+R)}{h \mu_{0} \omega(1+R)-(b-a) Z_{0} 2 \gamma(1+R)}, \\
\epsilon=\frac{[a+(b-a) \mu] 2 \gamma}{a \mu_{0} \epsilon_{0} \mu \omega^{2}}-\frac{(b-a)}{a},
\end{gathered}
$$

where $a$ is the half width of the sample, $b$ is the half width of the line, $Z_{0}$ is the characteristic impedance of the line, $\gamma$ is the propagation constant, $\omega$ is the angular frequency, and $R$ is the quantity given by

$$
\begin{gathered}
R=K_{1} \pm \sqrt{K_{1}^{2}-\frac{S_{11}}{S_{22}}} \\
K_{1}=\frac{S_{11} S_{22}-S_{12} S_{21}+1}{2 S_{22}}, \\
\gamma=\frac{j}{d} \ln \left(\frac{S_{21}}{1-R S_{22}}\right) .
\end{gathered}
$$

In contrast to the previous approach, the analytical character of relations (2) and (3) enables us to avoid the use of a numerical optimization procedure for the inverse problem. There are still some aspects to be considered in order to obtain more accurate results. First of all, our electromagnetic analysis takes into account only the air beside the sample but not the air gaps below and above it. In order to take into account these air gaps, the values of the retrieved permittivity and permeability are corrected using the Wiener's law. This mixing law is valid for thin gaps (thickness up to $500 \mu \mathrm{m})$. For the particular case when the sample and the central conductor have the same width $(\mathrm{a}=\mathrm{b})$, the expressions for $\epsilon$ and $\mu$ applying the air gaps correction are

$$
\begin{gathered}
\mu_{e f f}=\frac{\omega \mu_{0} h_{a}(1-R)-Z_{0} W \gamma(1+R)}{\omega h_{s} \mu_{0}(R-1)}, \\
\epsilon_{e f f}=\frac{h_{s} \gamma^{2}}{\omega^{2} \mu_{0} \epsilon_{0}\left(h_{s} \mu_{r}+h_{a}\right)-h_{a} \gamma^{2}},
\end{gathered}
$$

where $W$ is the width of the conductor, $h_{a}$ is the height of the air gap, and $h_{s}$ is the height of the sample to be characterized.

Finally, the intrinsic parameters of the material under test are obtained using the Hammerstad's model, ${ }^{12}$ which considers the part of energy that is propagated above the central conductor.

\section{MEASUREMENT}

S-parameters measurements of the test device were made using a Vectorial Network Analyzer (Agilent 8753ES) in the frequency range of $10 \mathrm{MHz}$ to $6 \mathrm{GHz}$. A specific calibration procedure based on short-open-load-thru (SOLT) is performed. The empty cell is inserted instead of the "Thru" standard during the calibration procedure in order to eliminate errors caused by the stripline (presence of tapers, attenuation, and impedance mismatch). Then, phases of measured S-parameters are corrected to place the reference's plane close to the sample's sides. 
At first, accuracy of the measurement method was evaluated by characterizing a known dielectric (Teflon, $\epsilon_{r}=2.1$ ) filling the transversal section of the line. Using S-parameters extracted from measurements, both analytical procedures (variational and transmission line approaches) were programmed using MathWorks ${ }^{\mathrm{TM}}$ MATLAB. Results are shown in Fig. 5. Good agreement between the two methods is observed and the permittivity value waited for Teflon shows that used analytical approaches are valid as well as the different approximations used for simplifying the models. Specifically with the transmission line theory based approach, air gaps and accurate effective medium models like Hammerstad allow us to correct the value of the real part of $\epsilon$.

For validating the measurement method for metamaterials, a sample provided by the IETR laboratory (University of Rennes 1, France) was characterized. It consists in a metasolenoid type structure, made of arrays of metallic split ring resonators (SRRs) printed on a dielectric substrate $\left(\epsilon_{r}=2.2\right)$ using multilayer technology (Fig. 6(a)). Dimensions of the unit cell are $1 \times 6 \times 1.57 \mathrm{~mm}$ and it is designed to operate at microwave frequencies, showing resonance around $5 \mathrm{GHz}$. The entire sample, composed by eleven unit cells in transversal direction is well suited for the stripline cell, filling the region between central strip conductor and ground plane (Fig. 6(b)). Measures were made between $10 \mathrm{MHz}$ and $6 \mathrm{GHz}$.

\section{RESULTS}

Measured S-parameters of the cell loaded with a metamaterial are shown in Fig. 7. Expected effects of resonance due to interactions between metallic inclusions and the incident electromagnetic wave appear around $5 \mathrm{GHz}$.

Permittivity and permeability spectra of metamaterial obtained from the different data processing programs are shown in Fig. 8. Experimental results are also compared with an electromagnetic simulation made with the software ANSYS HFSS ${ }^{\mathrm{TM}}$. The theoretical model of one unit cell is designed and delimited by perfect electric and magnetic conductors (PECs and PMCs) used as boundary conditions. Under these circumstances, the unit cell represents an infinite material. Simulated structure is excited by a plane wave and
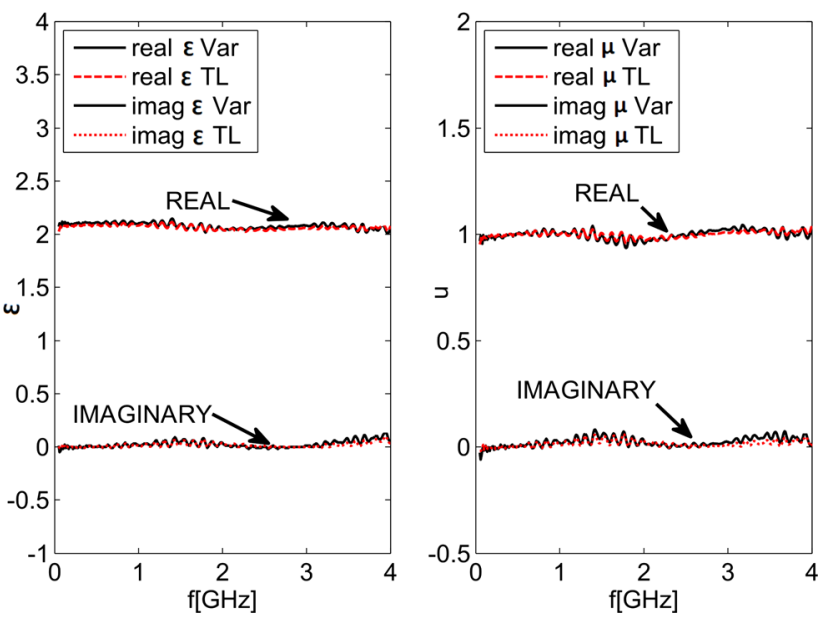

FIG. 5. Measured permittivity and permeability spectra (real and imaginary parts) of Teflon.

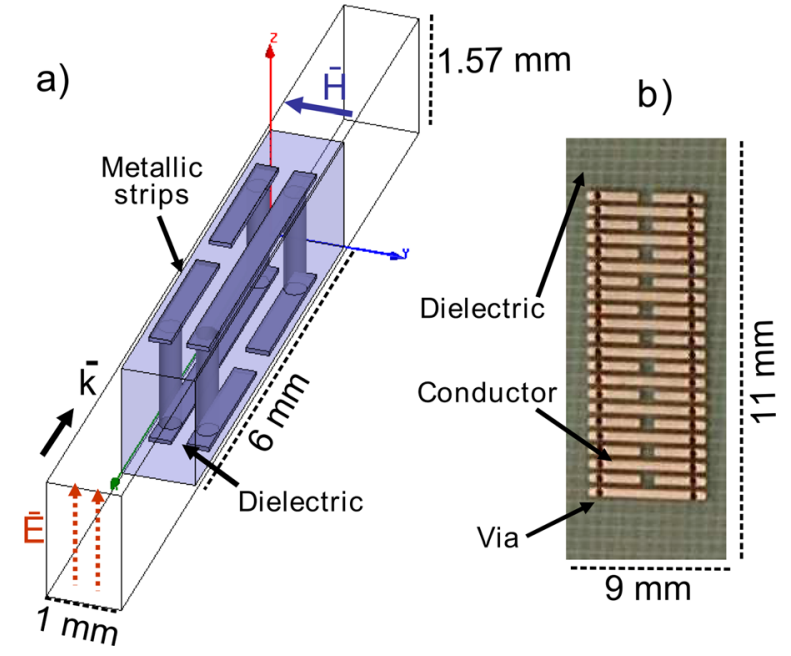

FIG. 6. Measured metasolenoid. (a) Unit cell. (b) Real sample (top view).

using robust NRW algorithm ${ }^{13}$ effective permittivity and permeability are calculated.

Experimental results demonstrate how expected resonant behavior of metamaterials can be fully characterized using the proposed technique. Good agreement is observed between the two retrieval methods and electromagnetic simulations. Using this method it is possible to obtain the behaviour of characteristic parameters, determining the resonant frequency band, its amplitude, and identifying negative and positive zones.

Nevertheless, some discrepancies in resonant region between simulation and measurements are noticed due to the following considerations. In first place, we assume some technological inaccuracies in the fabrication procedure of the sample like in rods metallisation process and wire alignment, as well as dimensions discrepancies between simulated and fabricated structure. In a second place, we have to consider the fact that in simulations we use a model representing
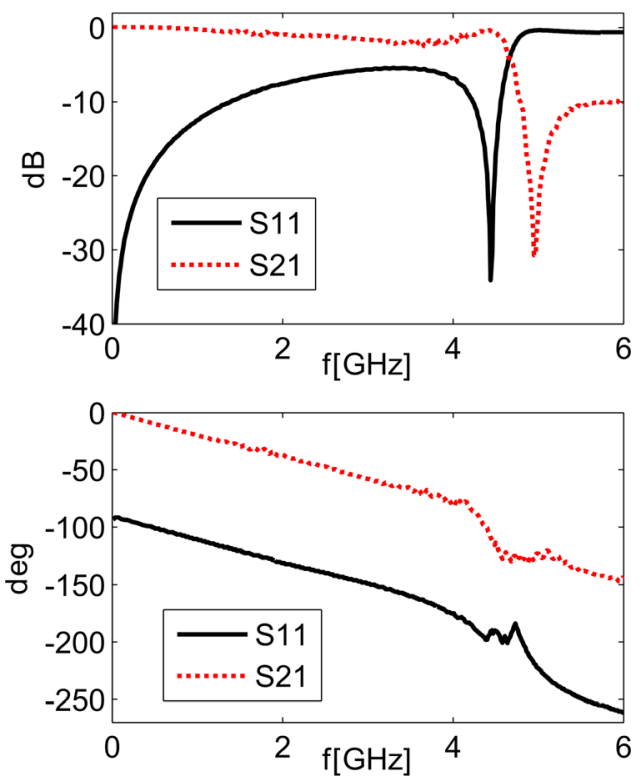

FIG. 7. Measured reflection and transmission coefficients of the stripline cell partially filled with metamaterial. Modules and phases. 

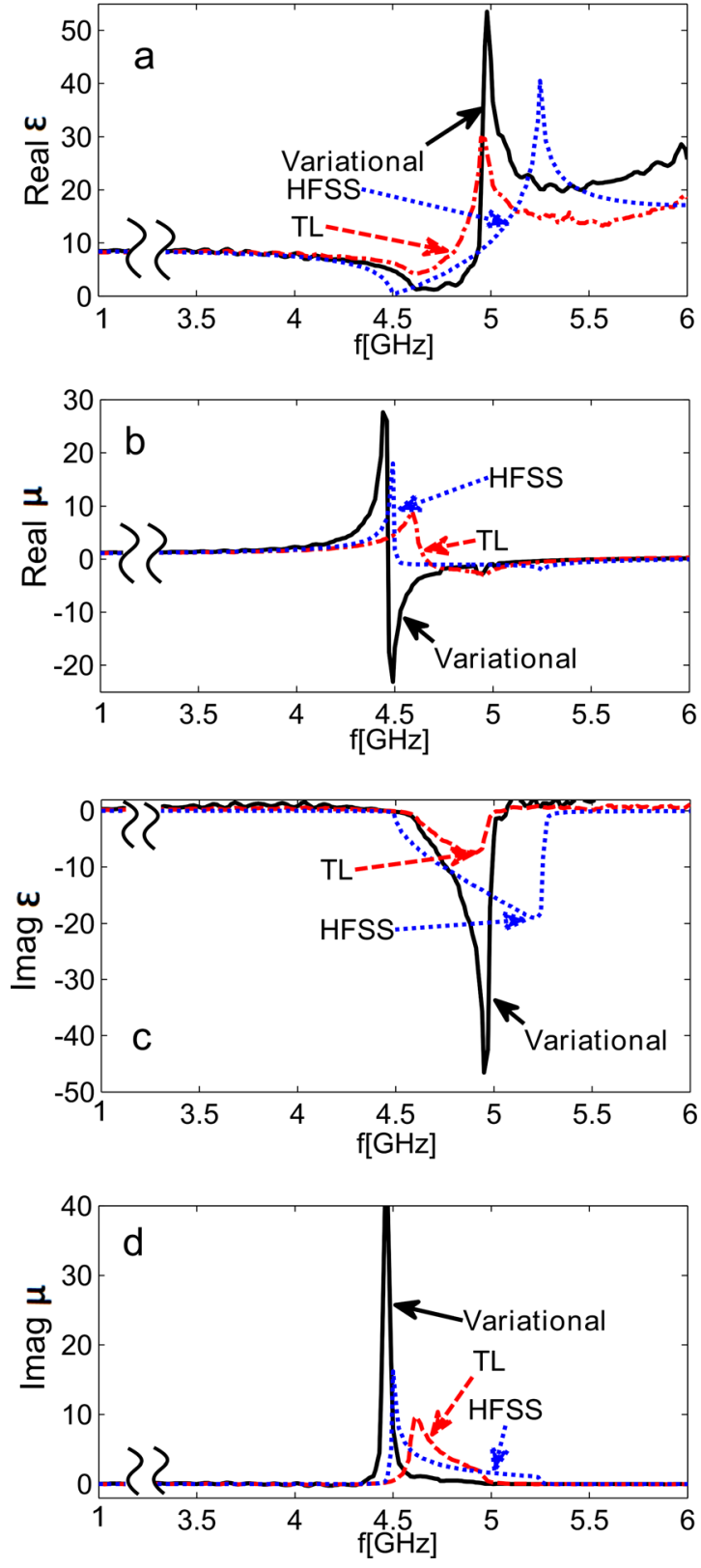

FIG. 8. Permittivity and permeability spectra. Comparison between simulated (ANSYS HFSS ${ }^{\text {TMM }}$ ) and measured data. (a) Real part of the permittivity spectrum. (b) Real part of the permeability spectrum. (c) Imaginary part of the permittivity spectrum. (d) Imaginary part of the permeability spectrum.

infinite number of cells while in measurement the number is limited (11 unit cells). In order to study the influence of the number of unit cells in our test sample, we have performed full wave simulations from commercial ANSYS HFSS ${ }^{\text {TM }}$ software. The experimental set up has been exactly reproduced and different scenarios have been simulated. We have increased the number of unit cells in various directions for inspecting the effect in S-parameters and retrieved constitutive parameters. Results were quite different for each case, however, a convergence of the permittivity and permeability values was observed as the number of cells increased. Thus, after a certain number of unit cells $(6,7)$ aligned in each direction, results converged to the one obtained from the simulation of an infinite structure. This convergence criteria can be satisfied experimentally in one direction ( $x$ axis, see
Fig. 1) thanks to the geometry of the stripline structure. For the two other directions, we are working out a new test fixture that will permit to change the distance between the strip and the ground plane of the stripline (y axis, see Fig. 1) to increase the number of measured unit cells. This new fixture will be also longer to study the influence of the number of unit cells along the propagation direction (z axis, see Fig. 1). In addition, we do not consider the edge effects due to the interaction between the unit cells of the metamaterial sample and the conductor planes of the stripline in the experimental procedure. Full-wave simulations also demonstrated that this edge effects can be minimized by increasing the number of unit cells along the y axis direction.

Last point may result from analytical approximations due to quasi-static theoretical approaches used to retrieve the electromagnetic parameters. We observe that measured and simulated magnitudes for the permittivity and the permeability are in very good agreement at non-resonant frequencies. On the other hand, in the vicinity of the resonant frequencies the magnitudes of the constitutive parameters are very high. This may produce electromagnetic interactions that cannot be described correctly with quasi-static theoretical approach. The domain of validity of this approach is strongly dependent of the permittivity and permeability values. That is why it is not surprising to observe a disagreement between theory and experiment in the vicinity of the resonances.

\section{CONCLUSION}

A method for measuring the characteristic parameters of planar metamaterials has been presented. The main advantage of the geometrical structure proposed for the measurement cell lies in the fact that the incident electromagnetic wave interacts homogeneously in magnitude and direction with all the inclusions composing the material, which reduces the possible inaccuracies due to the anisotropic character of this type of structures. Another advantage of this measurement method over existing techniques is the easiness of the experimental procedure, avoiding complicated and expensive set-ups such as large horn antennas and allowing the characterization of small samples in the decimeter wave range. Two different theoretical approaches for retrieving the intrinsic parameters of the material inside the cell have been tested showing a good grade of agreement between them. As a future work, the influence of (i) the number of unit cells that composes the sample under test and (ii) the metallic elements of the stripline over the behavior of the metamaterials will be studied. A new stripline fixture allowing us to minimize these effects will be presented.

${ }^{1}$ L. Chen, C. K. Ong, and B. T. G. Tan, IEEE Trans. Instrum. Meas. 48, 1023 (1999).

${ }^{2}$ H. Chen, J. Zhang, Y. Bai, Y. Luo, L. Ran, Q. Jiang, and J. A. Kong, Opt. Express 14, 12944 (2006)

${ }^{3}$ A. Erentok, P. Luljak, and R. Ziolkowski, IEEE Trans. Antennas Propag. 53, 160 (2005).

${ }^{4}$ L. Yousefi, M. Boybay, and O. Ramahi, IEEE Trans. Antennas Propag. 59, 1245 (2011).

${ }^{5}$ R. B. Greegor, C. G. Parazzoli, K. Li, B. E. C. Koltenbah, and M. Tanielian, Opt. Express 11, 688 (2003).

${ }^{6}$ Y. Hollander and R. Shavit, IET Proc. Microwaves, Antennas Propag. 5, 84 (2011). 
${ }^{7}$ E. Salahun, P. Quéffélec, M. Le Floc'h, and P. Gelin, IEEE Trans. Magn. 37, 2743 (2001).

${ }^{8} \mathrm{P}$. Quéffélec, S. Mallégol, and M. Le Floc'h, IEEE Trans. Microwave Theory Tech. 50, 2128 (2002).

${ }^{9}$ R. Crampagne, M. Ahmadpanah, and J. Guiraud, IEEE Trans. Microwave Theory Tech. 26, 82 (1978).
${ }^{10}$ A. M. Nicolson and G. F. Ross, IEEE Trans. Instrum. Meas. IM-19, 377 (1970). ${ }^{11}$ T. Coleman and Y. Li, SIAM J. Optim. 6, 418 (1996).

${ }^{12}$ E. Hammerstad and O. Jensen, in IEEE MTT-S Int. Microwave Symp. Dig. 1980, 407-409.

${ }^{13}$ X. Chen, T. Grzegorczyk, B. Wu, J. Pacheco, and J. Kong, Phys. Rev. E. 70, 016608 (2004). 